\title{
Short-Term Effect of Self-Selected Training Intensity on Ambulatory Blood Pressure in Hypertensive Older Women: A Randomized Controlled Trial
}

This article was published in the following Dove Press journal: Clinical Interventions in Aging

\author{
Júlio Sócrates (D) \\ Rodrigo Alberto Vieira \\ Browne iD' \\ Geovani \\ Araújo Dantas \\ Macêdo iD ${ }^{2}$ \\ Maria Beatriz Fonseca \\ Araújo (iD ${ }^{3}$ \\ Ronildo Paulo-Pereira (iD ${ }^{3}$ \\ Ludmila Lucena Pereira \\ Cabral (iD) \\ Bruno Erick Barros Lucena (D) \\ Luiz Fernando Farias-Junior (iD ${ }^{4}$ \\ Eduardo Caldas Costa (D) ${ }^{1-3}$ \\ 'Graduate Program in Health Sciences, \\ Federal University of Rio Grande do \\ Norte, Natal, Brazil; ${ }^{2}$ Graduate Program \\ in Physical Education, Federal University \\ of Rio Grande do Norte, Natal, Brazil; \\ ${ }^{3}$ Department of Physical Education, \\ Federal University of Rio Grande do \\ Norte, Natal, Brazil; ${ }^{4}$ Graduate Program \\ in Psychobiology, Federal University of \\ Rio Grande do Norte, Natal, Brazil
}

Correspondence: Eduardo Caldas Costa Department of Physical Education, Federal University of Rio Grande do Norte, BR I0I, Lagoa Nova, Natal, RN 59078-970, Brazil

Tel +558432153436

Fax +55843215345।

Email ecc@ufrnet.br
Purpose: To investigate the short-term effect of self-selected training intensity (SSTI) on ambulatory blood pressure (BP) in hypertensive older women.

Participants and Methods: This is a randomized, single-blind, two-arm, parallel-group controlled trial that included 40 medicated hypertensive older women (64.4 \pm 3.6 years; resting systolic $118 \pm 19$ and diastolic BP $68 \pm 9 \mathrm{mmHg}$ ). SSTI intervention was performed three times per week, $30-50$ minutes per session $(n=20)$. The control group participated in health education meetings once per week $(n=20)$. Ambulatory BP (primary outcome) and six-minute walking test performance (secondary outcome) were assessed at baseline and following 8 weeks of intervention. Heart rate (HR), rating of perceived exertion (RPE, 6-20), and affective valence (ie, feeling scale, $-5 /+5$ ) were recorded during all SSTI sessions. Intention-to-treat and per-protocol analyses were used for data analyses.

Results: Fifteen participants from the SSTI group and 17 from the control group completed the study. No differences in ambulatory BP (24-h, awake, and asleep) were observed between SSTI and control groups (intention-to-treat and per-protocol analyses; $p>0.05$ ). The SSTI group showed a greater six-minute walking test performance than the control group in the intention-to-treat and per-protocol analyses $(p<0.05)$. The participants exercised at $52 \pm 10 \%$ of HR reserve reported an RPE of $11 \pm 1$ and an affective valence of $3.4 \pm 1.1$ over the 8 -week period.

Conclusion: SSTI is a feasible approach to induce a more active lifestyle and increase health-related fitness in hypertensive older women, although it does not improve BP control over a short-term period.

Keywords: exercise, hypertension, aging

\section{Introduction}

Hypertension is a major risk factor for premature mortality worldwide, being mainly associated with cardiovascular diseases. ${ }^{1}$ In the last four decades, the number of adults with hypertension increased from 594 million in 1975 to 1.1 billion in 2015. The increase of hypertension occurred mainly in low-income and middle-income countries. $^{2}$ In addition, the hypertension care cascade (ie, hypertension awareness, treatment, and control) was worse in low- and middle-income than in high-income countries. $^{3}$ It should be noted that the prevalence of hypertension is higher in older adults, ${ }^{1}$ especially in older women. ${ }^{4,5}$ Therefore, improvement in the hypertension care cascade is needed, especially at the blood pressure (BP) control level, in order to attenuate the risk of premature cardiovascular events and mortality. 
Physical exercise is a cornerstone of non-pharmacological approaches to reduce BP and improve hypertension control. ${ }^{6,7}$ A recent network meta-analysis showed that the systolic BPlowering effect of exercise training appears to be similar to antihypertensive medications among individuals with hypertension. ${ }^{8}$ Despite the well-known antihypertensive effect of regular exercise training, the SABE Survey (Health, Welfare and Aging Survey), which is a home-focused epidemiological study in Brazil, revealed that $71.4 \%$ of hypertensive older adults are inactive; ie, $<150$ minutes per week of moderate physical activity, and $<75$ minutes per week of vigorous physical activity. ${ }^{9}$ Thus, effort should be made to increase the participation and adherence of hypertensive older adults in regular physical exercise.

The traditional exercise prescription recommended by clinical hypertension guidelines have focused on the FITT principle (Frequency, Intensity, Time, and Type), ${ }^{6,7}$ using heart rate- (HR) and oxygen uptake-based $\left(\mathrm{VO}_{2}\right)$ approaches for exercise intensity prescription and monitoring. However, these methods are not accessible or easy to understand for most hypertensive older adults. It has been argued that self-selected exercise intensity may be an interesting option to increase participation and adherence to regular physical exercise, ${ }^{10}$ mainly due to its psychological advantages such as higher perceived autonomy, selfefficacy, affective response, and lower perceived exertion. ${ }^{11-13}$ Self-selected exercise intensity is characterized when individuals choose their own exercise intensity according to their preferences. ${ }^{10}$ Older adults typically self-select an exercise intensity close to their ventilatory threshold, ${ }^{10,14}$ which is within the recommended range proposed for hypertensive patients. For example, the American College of Sports Medicine recommends an exercise intensity between $40 \%$ and $59 \%$ of $\mathrm{VO}_{2}$ reserve or HR reserve for hypertensive individuals. ${ }^{7}$

Two previous studies from our group showed that hypertensive older women self-select an exercise intensity between $50 \%$ and $60 \%$ of HR reserve and report the exercise sessions as "light" and "pleasant". ${ }^{15,16}$ In addition, Costa et al ${ }^{16}$ showed that a single 30-minute self-selected exercise intensity session elicited a reduction in ambulatory systolic (BP) in inactive hypertensive older women. The systolic postexercise hypotension (PEH) of $\sim 6 \mathrm{mmHg}$ lasted up to a sixhour period and contributed to a reduction in the mean values of systolic BP in the 20-hour and awake periods. These results suggest that the self-selected exercise intensity has the potential to induce a BP-lowering effect following a regular exercise training period. ${ }^{17}$ However, to the best of our knowledge, there is a scarcity of studies investigating the chronic effect of a self-selected training intensity (SSTI) intervention on BP and fitness-related outcomes in hypertensive older adults. Therefore, this study investigated the shortterm chronic effect of a SSTI intervention on ambulatory BP (primary outcome) and six-minute walking test performance (secondary outcome) in inactive hypertensive older women. It was hypothesized that 8 weeks of SSTI would reduce ambulatory BP and improve the six-minute walking test performance in these individuals.

\section{Participants and Methods Trial Design}

This is a randomized, single-blind, two-arm, parallel-group controlled trial designed to investigate the short-term chronic effect of a SSTI intervention on ambulatory BP in hypertensive older women. This trial is reported in accordance with the CONSORT statement guidelines. ${ }^{17}$ All procedures related to this study were conducted at the Department of Physical Education and at the University Hospital Onofre Lopes from the Federal University of Rio Grande do Norte, Natal, RN, Brazil, between September 2018 and November 2019. All participants were informed about the study procedures and provided written informed consent. The study was carried out in line with the Declaration of Helsinki and was approved by the Institutional Ethics Committee (protocol 2.840.999/2018). The study was also registered in the Brazilian Clinical Trials Registry (http:// ensaiosclinicos.gov.br/; ID: RBR-3h5394).

\section{Participants}

A total of 40 community-dwelling hypertensive older women were included in this study (Figure 1). All participants were recruited from Natal, RN, Brazil, by advertisements on radio, flyers, and e-flyers in social media (WhatsApp $^{\circledR}$, Instagram $^{\circledR}$ and Facebook ${ }^{\circledR}$ ). The inclusion criteria for the participants were: i) aged $60-75$ years; ii) previous diagnosis of hypertension according to the Brazilian guidelines on hypertension; ${ }^{18}$ iii) currently taking antihypertensive medication(s); iv) body mass index (BMI) $<35 \mathrm{~kg} / \mathrm{m}^{2}$; v) being inactive; ie, less than 600 $\mathrm{MET} / \mathrm{min} /$ week of physical activities ${ }^{19}$ and $<7500$ steps per day; ${ }^{20}$ vi) no prior history of major cardiovascular disease or cardiovascular events (eg, acute myocardial infarction, stroke, coronary artery disease, arrhythmias, or peripheral vascular disease); vii) no osteomioarticular injury which limited the ability to perform exercise. The 


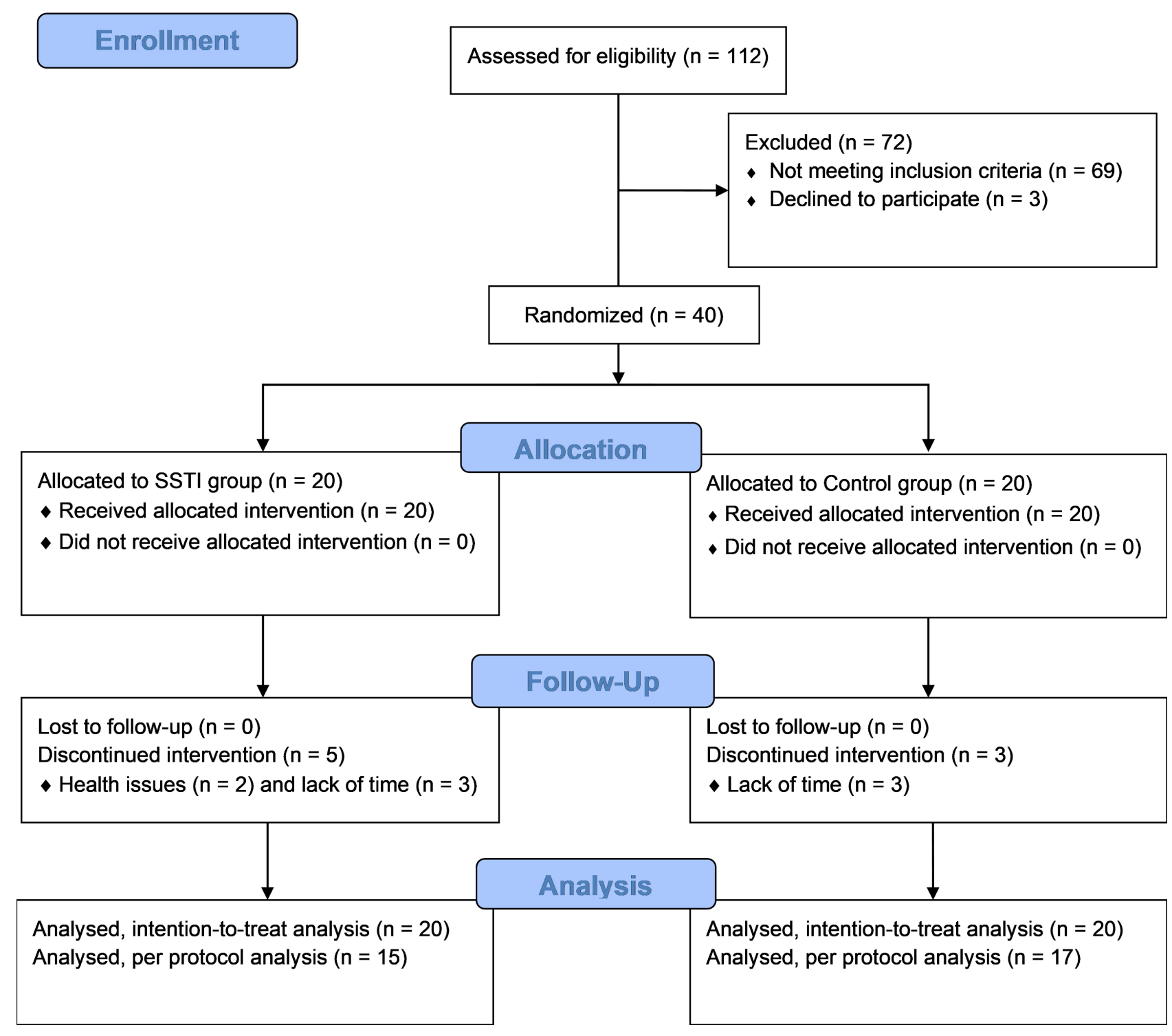

Figure I Study flowchart.

Abbreviation: SSTI, self-selected training intensity group.

exclusion criteria were: i) having pain or injury which limits their ability to exercise during the study; ii) changes in antihypertensive medication(s) during the study.

\section{Procedures}

All participants were screened at their first visit using the Physical Activity Readiness Questionnaire, ${ }^{21}$ medical history, medication use, and the initial physical activity level assessment using an adapted version of the Minnesota Leisure Time Activities Questionnaire. ${ }^{19}$ A clinical examination provided body weight $(\mathrm{kg})$, height $(\mathrm{m})$, resting HR (bpm), and resting BP $(\mathrm{mmHg})$ measurements. BMI was calculated as weight $(\mathrm{kg})$ divided by height $(\mathrm{m})$ squared. Resting HR was measured after 10 minutes of rest in a supine position in a calm and controlled room $\left(24-26^{\circ} \mathrm{C}\right)$ using an HR monitor (Polar Electro ${ }^{\circledR}$, Kempele, Finland). Resting BP was measured in a seated position using an oscillometric device $\left(\mathrm{Omron}^{\circledR}\right.$ HEM-780-E, Kyoto, Japan) in triplicate with 1-minute intervals between each measure. The average value of the last two measures was recorded. In the second visit, the participants performed the maximal graded exercise test with a cardiologist in order to exclude symptoms and electrocardiographical alterations suggestive of heart disease. In the third visit, the ambulatory BP of the participants during a 24-hour period was assessed. The participants then returned to the laboratory on the next day to remove the ambulatory BP monitoring device (CardioMAPA, Cardios ${ }^{\circledR}$, Brazil) and to perform the sixminute walking test. Afterward, participants were fitted with 
a wrist pedometer (Omron ${ }^{\circledR}$ HJ-321, Kyoto, Japan) to confirm their inactive physical activity level as assessed by the number of steps accumulated per day over a 1-week period. The participants returned to the laboratory 1 week later to remove the wrist pedometer and were randomly allocated to the SSTI or control group.

\section{Physical Activity Level}

An adapted version of the Minnesota Leisure Time Activities Questionnaire ${ }^{19}$ was used to identify inactive participants in the screening visit. Afterward, those identified as inactive (ie, $<600 \mathrm{MET} / \mathrm{min} /$ week) were selected for the pedometer-measured physical activity level assessment in order to confirm their inactive status. Pedometers (Omron ${ }^{\circledR}, \mathrm{HJ}-321$ Tri-Axis Alvita, USA) were individually adjusted for the participants based on their stride length, weight and height according to the manufacturer's instructions. A total number of steps per day less than 7500 was used as a criterion to determine participants as inactive. $^{20}$ The participants returned to the laboratory 1 week after the fourth screening visit to remove the wrist pedometer. Only participants classified as inactive in the Minnesota Leisure Time Activities Questionnaire ${ }^{19}$ and who showed less than 7500 steps per day in the pedometer-measured physical activity level assessment were included in the study.

\section{Maximal Graded Exercise Test}

The participants performed a maximal graded exercise test on a motorized treadmill (Centurion 200, Micromed ${ }^{\circledR}$, Brazil) to determine their $\mathrm{HR}_{\max }$ and to exclude symptoms and electrocardiographical alterations suggestive of heart disease. Participants were continuously monitored with 12-lead electrocardiography by a cardiologist during the maximal graded exercise test and during a five-minute recovery period. An incremental ramp protocol was performed by the participants starting at a speed of $2.5 \mathrm{~km} / \mathrm{h}$ and $4 \%$ grade with continuous increments to reach $5.5 \mathrm{~km} /$ $\mathrm{h}$ and $14 \%$ grade at 10 minutes. The BP was measured every 2 minutes during and immediately following the end of the maximal graded exercise test by the auscultatory method. RPE was recorded every minute using Borg's RPE scale (6-20). ${ }^{22}$ The graded exercise test was considered maximal when the participant reached an RPE $>17$ and HR within $\pm 10 \mathrm{bpm}$ of age-predicted $\mathrm{HR}_{\max }$ (220age) ${ }^{23}$ For participants using a $\beta$-blocker, only RPE was used as a criterion of volitional exhaustion. Participants performed the maximal graded exercise test under their regular antihypertensive medication.

\section{Six-Minute Walking Test}

The six-minute walking test (6MWT) was conducted according to the procedures established by Rikli and Jones $^{24}$ and was used as a proxy of cardiorespiratory fitness. ${ }^{25}$ A previous study showed that the walking distance covered in the 6MWT predicted maximal oxygen uptake $\left(R^{2}=0.76\right)$ obtained from a maximal cardiopulmonary test, which is a "gold-standard" method to assess cardiorespiratory fitness. ${ }^{26}$ Therefore, we used the 6MWT as a proxy of cardiorespiratory fitness due to its feasibility and potential to provide valuable information in clinical practice. $^{25}$

\section{Ambulatory Blood Pressure Monitoring}

Participants wore an ambulatory BP device (CardioMAPA, Cardios $^{\circledR}$, Brazil) on their non-dominant arm according to the Brazilian guidelines for ambulatory BP monitoring ${ }^{27}$ over a 24-hour period. The device was programmed to measure BP every 15 minutes during their awake and every 30 minutes during their sleeping periods. A minimum of 16 and eight BP measurements during awake and sleeping periods, respectively, had to be successfully recorded to be included in the analysis. Participants came back to the laboratory the following morning after each session so that the BP information could be downloaded to a computer. Data was recorded during the awake and sleeping periods defined according to each participant. The average values for each period were considered for data analysis (ie, 24-hour, awake, and asleep). In addition, BP load was calculated during the 24hour period. This index is defined as the percentage of readings that exceeds the normal BP values in both awake and asleep periods. Systolic BP $\geq 135$ and diastolic BP $\geq$ $85 \mathrm{mmHg}$ were considered abnormal in the awake period, while systolic $\mathrm{BP} \geq 120$ and diastolic $\mathrm{BP} \geq 70 \mathrm{mmHg}$ were considered abnormal in the asleep period. All procedures related to the ambulatory BP monitoring were explained to the participants at the health screening visit based on the Brazilian guidelines for ambulatory BP monitoring. ${ }^{27}$

\section{Self-Selected Training Intensity Intervention}

The SSTI occurred three times per week over 8 weeks. The duration of the SSTI sessions was increased from 
week one (30 minutes) to week five (50 minutes); ie, 5 minutes per week. The SSTI sessions had 50 minutes duration from week five to week eight. All SSTI sessions were performed in the afternoon between 4:00 and 6:00 p.m. on a standard outdoor $400 \mathrm{~m}$ track. Participants' BP was measured immediately prior to each SSTI session. They were allowed to exercise if their BP was lower than $160 / 105 \mathrm{mmHg}^{23}$ Participants were instructed to regulate their own pace before the SSTI sessions using the following statement and following the same procedures of previous studies ${ }^{15,16}$ from our group: We would like that you exercise for $[30,35,40,45$, or 50 minutes] at a pace of your own choosing. You can freely change the pace at any time during the exercise. Participants performed a stretching routing and then warmed up for $5 \mathrm{~min}$ utes to adjust their pace before starting the SSTI session. Participants then cooled down afterwards for 5 minutes by walking at a slow pace. All participants were fitted with a HR monitor (Polar Electro ${ }^{\circledR}$, Kempele, Finland) to monitor HR responses during SSTI sessions. In addition, whole-body perceived exertion using Borg's RPE scale $(6-20)^{22}$ and affective valence using the Feeling Scale $(-5 /+5)^{28}$ were reported by participants every 10 minutes during the SSTI sessions over the eight-week period of intervention. All participants were individually instructed about the use of the Borg's RPE scale and Feeling Scale prior to beginning the SSTI intervention. RPE was defined as subjective intensity of effort, strain, and/or fatigue felt during exercise. ${ }^{22}$ Low and high perceptual anchors for Borg's RPE scale were established during the maximal graded exercise test. A rating of 6 (low anchor, "very, very light") was assigned to the lowest exercise intensity, while a rating of 20 (high anchor, "very, very hard") was assigned to the highest exercise intensity. The Feeling Scale is an 11-point bipolar scale ranging from +5 to -5 . This scale presents verbal anchors: $-5=$ very bad; $-3=$ bad; $-1=$ fairly bad; $0=$ neutral; +1 fairly good; $+3=$ good; and $+5=$ very good. The participants received standard instructions explaining the Feeling Scale according to Hardy and Rejeski. ${ }^{28}$ All SSTI sessions were performed individually in order to avoid interference on the self-selected pace, RPE and affective valence.

\section{Control Group}

The control group participated in group-based health education meetings once per week over the 8 weeks of intervention at the Department of Physical Education, Federal University of Rio Grande do Norte. Every education meeting had 60 minutes of duration and was conducted by two researchers (LLPC, MBFA). The topics of the education meetings covered basic knowledge about selfcare in aging, healthy and unhealthy behaviors (eg hydration, sleep, alcohol intake, sedentary time), and prevention and management of non-communicable diseases (eg, hypertension, type 2 diabetes, signals and symptoms of cardiovascular events). Moreover, group dynamics, ludic activities and board games were also conducted during the meetings. The comparator of the SSTI intervention was health education because this is a usual care approach in public primary care settings in Brazil for hypertensive adults, in addition to the pharmacological treatment.

\section{Sample Size and Randomization}

Previous meta-analyses showed that a decrease of $5 \mathrm{mmHg}$ in systolic BP provides a clinically meaningful risk reduction in cardiovascular diseases and mortality. ${ }^{29,30}$ A metaanalysis conducted by Sosner et $\mathrm{al}^{31}$ showed that aerobic exercise training reduces 24-hour ambulatory BP by $4 \mathrm{mmHg}$; however, the magnitude of the BP-lowering effect is higher in individuals with pre- to established hypertension (ie, resting $\mathrm{BP} \geq 130 / 85 \mathrm{mmHg}$ ). Based on these previous studies, ${ }^{29-31}$ a priori sample size calculation was conducted to provide sufficient power (1- $\beta$ of $80 \%$ and alpha of $5 \%$ ) for a reduction of $5 \pm 5 \mathrm{mmHg}$ in 24-hour ambulatory BP (primary outcome) in the SSTI group compared to the control group. A total of 34 participants were needed to fulfill the study power $\left(\mathrm{G}^{*}\right.$ Power software, version 3.1.9.2). Thus, considering a dropout rate of $20 \%, 40$ participants were recruited for the study. After all baseline assessments, a computer-based simple randomization (www.graphpad.com) was carried out to determine the allocation of the participants to the SSTI $(n=20)$ or the control group $(n=20)$. This procedure was conducted by an independent researcher (ECC) not involved in the recruitment, allocation, assessment (baseline and following 8 weeks), and SSTI intervention or health education meetings. Afterward, the 40 sequential codes from the randomization list were included in 40 opaque and closed envelopes. Thus, allocation concealment was implemented. In addition, blinded researchers (RAVB, LFFJ, GADM) conducted all procedures related to the assessments at baseline and following 8 weeks of intervention.

\section{Statistical Analysis}

Data normality was tested using the Shapiro-Wilk test, skewness and kurtosis (score-z: -1.96 to +1.96 ). Results 
are expressed as mean $\pm \mathrm{SD}$ and absolute and relative frequency. An independent $t$-test was used to compare the mean values of resting and ambulatory BP, 6MWT, pedometer-measured physical activity level, and anthropometric measures at baseline. The Friedman test was used to compare HR reserve, RPE and affective valence during the SSTI sessions over the 8 weeks of intervention. Intention-to-treat and per-protocol analyses were conducted for both primary and secondary outcomes. A generalized estimating equation (GEE) model was used to assess the group (2 factors: SSTI vs control) by time ( 2 factors: baseline vs eight weeks) interaction on primary (ambulatory BP) and secondary (6MWT performance) outcomes. All GEE models used the gamma distribution with an identity link based on the goodness of fit, except for the BP load, which used a tweedie distribution with identity link. The goodness of fit of the models was verified by the normal Q-Q plot and Akaike information criterion. A two-tailed $\mathrm{p}<0.05$ was considered statistically significant for all analyses. Data analysis was performed using IBM SPSS Statistics for Win/v.25.0 (IBM Corp., Armonk, NY). Cohen's $\mathrm{d}_{\mathrm{s}}$ for comparisons betweengroups and Cohen's $d_{z}$ for comparisons within-group were used to calculate the effect size (ES). ${ }^{32}$ The following criteria for the interpretation of the ES were adopted: $<0.50$, small; 0.50 to 0.79 , medium; and $\geq 0.80$, large. $^{33}$

\section{Results}

Eight participants dropped out of the study (ie, five from the SSTI group and three from the control group). Six participants reported a lack of time to continue the study, one participant had a renal calculi crisis not related to the study, and one was excluded because they reported having knee pain in the second SSTI session. No more adverse events occurred over the 8 weeks of the SSTI intervention. The attendance rate of the SSTI sessions was $91.7 \%$. Table 1 shows the characteristics of the participants included in the study. The participants had $9.4 \pm 5.9$ years of hypertension diagnosis. Regarding the antihypertensive medication use, $45 \%(\mathrm{n}=18)$ used only one medication, $47 \%(n=19)$ two medications, and $8 \%(n=3)$ three or more medications. A total of 10 Caucasian, 8 Pardo, and 2 Black individuals were included in the SSTI group and 9 Caucasian, 7 Pardo, and 4 Black individuals were included in the control group. No differences were observed for the pedometer-measured physical activity level between the groups at baseline $(4213 \pm 1665$ vs $4603 \pm 1520$ steps/ day; $\mathrm{p}=0.808$; Cohen's $\left.\mathrm{d}_{\mathrm{s}}=0.24\right)$.
Table I Characteristics of the Self-Selected Training Intensity and Control Groups

\begin{tabular}{|l|l|l|l|}
\hline Variables & SSTI & Control & P-value \\
\hline $\mathrm{N}$ & 20 & 20 & \\
Age (years) & $64.7 \pm 3.3$ & $64.2 \pm 3.9$ & 0.359 \\
Weight (kg) & $67.3 \pm 11.8$ & $73.4 \pm 15.4$ & 0.110 \\
Height (cm) & $153.4 \pm 4.9$ & $153.7 \pm 6.1$ & 0.342 \\
Body Mass Index (kg/m²) & $29.1 \pm 5.6$ & $31.1 \pm 6.1$ & 0.340 \\
Six-Minute Walking Test (m) & $467 \pm 45$ & $465 \pm 67$ & 0.710 \\
Resting SBP (mmHg) & $122 \pm 20$ & $114 \pm 16$ & 0.435 \\
Resting DBP (mmHg) & $70 \pm 10$ & $65 \pm 8$ & 0.884 \\
Resting Heart Rate (bpm) & $71 \pm 7$ & $74 \pm 9$ & 0.440 \\
Maximal Heart Rate (bpm) & $151 \pm 16$ & $142 \pm 12$ & 0.192 \\
Heart Rate Reserve (bpm) & $80 \pm 17$ & $68 \pm 14$ & 0.558 \\
\hline Antihypertensive medications, & & & \\
n (\%) & & & \\
$\quad$ Calcium Channel Blocker & $2(10 \%)$ & $2(10 \%)$ & \\
Diuretics & $7(35 \%)$ & $7(35 \%)$ & \\
Angiotensin Receptor & $12(60 \%)$ & $18(90 \%)$ & \\
Antagonists & & & \\
Beta-Blocker & $5(25 \%)$ & $4(20 \%)$ & \\
ACE Inhibitor & $7(35 \%)$ & $1(5 \%)$ & \\
\hline
\end{tabular}

Note: Values expressed as mean \pm SD or absolute and relative frequency (\%). Abbreviations: ACE, angiotensin converting enzyme; DBP, diastolic blood pressure; SBP, systolic blood pressure; SSTI, self-selected training intensity.

Table 2 shows the HR, RPE, and affective valence during the SSTI sessions over the 8 weeks of intervention. No differences were found over the 8 weeks of intervention in the HR, RPE and affective valence $(p>0.05)$. Participants exercised at an average of $52 \pm 10 \%$ of HR reserve, reported an RPE of $11 \pm 1$ and an affective valence of $3.4 \pm 1.1$.

Table 3 shows the values of ambulatory BP at baseline and following the 8 weeks of intervention in the SSTI and control groups. Both intention-to-treat $(n=40)$ and perprotocol $(\mathrm{n}=32)$ analyses revealed no significant differences in systolic and diastolic 24-hour, awake, and asleep BP between the SSTI and control groups $(p>0.05)$. There was no difference in systolic 24-hour BP load in the SSTI compared to the control group, both in the intention-totreat analysis $(\mathrm{p}=0.079)$ and per-protocol analysis $(\mathrm{p}=0.076)$.

Figure 2 shows the individual 24-hour BP pre-post changes of participants with uncontrolled (ie, systolic BP $\geq 130 \mathrm{mmHg}$; panel A) and controlled (ie, systolic BP < $130 \mathrm{mmHg}$; panel B) ambulatory BP levels at baseline based on per-protocol analysis. All participants from the SSTI group with uncontrolled BP levels showed a reduction in the 24-hour systolic BP (from $\sim 2$ to $\sim 6$ 
Table 2 Heart Rate, Rating of Perceived Exertion and Affective Valence During the Self-Selected Training Intensity Sessions Over the Eight Weeks of Intervention

\begin{tabular}{|c|c|c|c|c|c|c|c|c|c|}
\hline & \multicolumn{8}{|c|}{ Self-Selected Training Intensity - Weeks } & \multirow[t]{2}{*}{ P-value } \\
\hline & $\mathbf{I}$ & 2 & 3 & 4 & 5 & 6 & 7 & 8 & \\
\hline HR Reserve (\%) & $55 \pm 13$ & $52 \pm 14$ & $54 \pm 12$ & $54 \pm 12$ & $54 \pm 11$ & $55 \pm 12$ & $52 \pm 13$ & $57 \pm 12$ & 0.953 \\
\hline RPE (6-20) & $\mathrm{II} \pm \mathrm{I}$ & $\mathrm{II} \pm \mathrm{I}$ & $11 \pm 1$ & $\mathrm{II} \pm \mathrm{I}$ & $\mathrm{II} \pm \mathrm{I}$ & $\mathrm{II} \pm \mathrm{I}$ & $\mathrm{II} \pm \mathrm{I}$ & $\mathrm{II} \pm \mathrm{I}$ & 0.999 \\
\hline Affect $(-5 /+5)$ & $3.3 \pm 0.8$ & $3.2 \pm 1.2$ & $3.2 \pm 0.8$ & $3.2 \pm 0.9$ & $3.2 \pm 0.8$ & $3.3 \pm 1.1$ & $3.6 \pm 0.9$ & $3.5 \pm 1.0$ & 0.173 \\
\hline
\end{tabular}

Note: Values expressed as mean \pm SD.

Abbreviations: HR, heart rate; RPE, rating of perceived exertion.

Table 3 Ambulatory BP at Baseline and Following Eight Weeks of Intervention in the Self-Selected Training Intensity and Control Groups

\begin{tabular}{|c|c|c|c|c|c|c|c|c|}
\hline \multirow[t]{2}{*}{ Ambulatory BP } & \multicolumn{2}{|c|}{$\begin{array}{l}\text { SSTI } \\
\text { ITT }(n=20) ; \text { PP }(n=15)\end{array}$} & \multirow[t]{2}{*}{ ES } & \multicolumn{2}{|c|}{$\begin{array}{l}\text { Control } \\
\text { ITT }(n=20) ; \text { PP }(n=\mid 7)\end{array}$} & \multirow[t]{2}{*}{ ES } & & \multirow[t]{2}{*}{ P-value } \\
\hline & Baseline & 8 Week & & Baseline & 8 Week & & $\beta(95 \%$ Wald Cl) & \\
\hline \multicolumn{9}{|l|}{ 24-hour } \\
\hline \multicolumn{9}{|l|}{ Intention-to-Treat } \\
\hline $\mathrm{SBP}(\mathrm{mmHg})$ & $124 \pm 16$ & $123 \pm 15$ & 0.19 & $123 \pm 16$ & $124 \pm 17$ & 0.14 & $-2.3(-6.2$ to 1.5$)$ & 0.240 \\
\hline $\mathrm{DBP}(\mathrm{mmHg})$ & $69 \pm 8$ & $68 \pm 7$ & 0.25 & $67 \pm 7$ & $67 \pm 8$ & 0.00 & $-1.2(-3.5$ to 1.2$)$ & 0.333 \\
\hline \multicolumn{9}{|l|}{ Per protocol } \\
\hline $\mathrm{SBP}(\mathrm{mmHg})$ & $122 \pm 14$ & $121 \pm 12$ & 0.16 & $122 \pm 16$ & $124 \pm 18$ & 0.25 & $-2.8(-7.6$ to 1.9$)$ & $0.24 I$ \\
\hline $\mathrm{DBP}(\mathrm{mmHg})$ & $69 \pm 8$ & $67 \pm 8$ & 0.46 & $67 \pm 6$ & $67 \pm 8$ & 0.00 & $-1.5(-4.5$ to 1.5$)$ & 0.298 \\
\hline \multicolumn{9}{|l|}{ Awake } \\
\hline \multicolumn{9}{|l|}{ Intention-to-Treat } \\
\hline $\mathrm{SBP}(\mathrm{mmHg})$ & $125 \pm 16$ & $125 \pm 15$ & 0.00 & $124 \pm 15$ & $126 \pm 17$ & 0.25 & $-2.0(-6.1$ to 2.0$)$ & 0.326 \\
\hline $\mathrm{DBP}(\mathrm{mmHg})$ & $71 \pm 8$ & $70 \pm 8$ & 0.25 & $68 \pm 7$ & $69 \pm 5$ & 0.00 & $-0.6(-3.0$ to 1.8$)$ & 0.626 \\
\hline \multicolumn{9}{|l|}{ Per protocol } \\
\hline $\mathrm{SBP}(\mathrm{mmHg})$ & $123 \pm 14$ & $123 \pm 12$ & 0.00 & $124 \pm 16$ & $126 \pm 18$ & 0.23 & $-2.4(-7.4$ to 2.6$)$ & 0.344 \\
\hline $\mathrm{DBP}(\mathrm{mmHg})$ & $70 \pm 9$ & $70 \pm 8$ & 0.00 & $69 \pm 7$ & $69 \pm 8$ & 0.00 & $-0.8(-3.8$ to 2.3$)$ & 0.615 \\
\hline \multicolumn{9}{|l|}{ Asleep } \\
\hline \multicolumn{9}{|l|}{ Intention-to-Treat } \\
\hline $\mathrm{SBP}(\mathrm{mmHg})$ & $119 \pm 15$ & $116 \pm 14$ & 0.45 & $119 \pm 18$ & $119 \pm 18$ & 0.00 & $-2.8(-7.4$ to 1.9$)$ & 0.249 \\
\hline $\mathrm{DBP}(\mathrm{mmHg})$ & $63 \pm 8$ & $60 \pm 7$ & 0.66 & $61 \pm 7$ & $61 \pm 9$ & 0.00 & $-2.0(-5.3$ to 1.2$)$ & 0.215 \\
\hline \multicolumn{9}{|l|}{ Per protocol } \\
\hline $\mathrm{SBP}(\mathrm{mmHg})$ & $118 \pm 14$ & $115 \pm 11$ & 0.38 & $118 \pm 18$ & $118 \pm 18$ & 0.00 & $-3.6(-9.3$ to 2.1$)$ & 0.219 \\
\hline $\mathrm{DBP}(\mathrm{mmHg})$ & $63 \pm 9$ & $59 \pm 7$ & 0.76 & $61 \pm 7$ & $60 \pm 9$ & 0.15 & $-2.8(-6.7$ to I.I) & 0.158 \\
\hline \multicolumn{9}{|l|}{ 24-hour BP Load } \\
\hline \multicolumn{9}{|l|}{ Intention-to-Treat } \\
\hline SBP (\%) & $33 \pm 34$ & $30 \pm 31$ & 0.28 & $30 \pm 33$ & $34 \pm 35$ & 0.30 & $-6.5(-13.7$ to 0.7$)$ & 0.079 \\
\hline DBP (\%) & $15 \pm 22$ & $12 \pm 16$ & 0.23 & $12 \pm 15$ & $13 \pm 17$ & 0.10 & $-3.5(-9.3$ to 2.3$)$ & 0.237 \\
\hline \multicolumn{9}{|l|}{ Per protocol } \\
\hline SBP (\%) & $30 \pm 32$ & $26 \pm 28$ & 0.33 & $28 \pm 32$ & $33 \pm 34$ & 0.35 & $-8.1(-17.0$ to 0.9$)$ & 0.076 \\
\hline DBP (\%) & $14 \pm 25$ & $11 \pm 16$ & 0.28 & $11 \pm 13$ & $12 \pm 16$ & 0.09 & $-4.5(-11.7$ to 2.7$)$ & 0.223 \\
\hline
\end{tabular}

Notes: Values expressed as mean \pm SD and parameter estimates $(\beta)$ and $95 \%$ Wald confidence interval $(\mathrm{Cl})$. ES: Cohen's $d_{z}$ effect size $(<0.50$, small; 0.50 to 0.79 , medium; and $\geq 0.80$, large).

Abbreviations: BP, blood pressure; DBP, diastolic blood pressure; ITT, intention-to-treat analysis; PP, per-protocol analysis; SBP, systolic blood pressure; SSTI, self-selected training intensity group.

$\mathrm{mmHg}$ ). Considering the five participants from the control group with uncontrolled BP levels, one did not change the 24-hour systolic BP, two showed an increase ( $\sim 7$ and $\sim 14$
$\mathrm{mmHg}$ ), and two showed a decrease ( $\sim 9$ and $\sim 3 \mathrm{mmHg})$. Regarding the participants from the SSTI group with controlled BP levels at baseline, $63.7 \%(\mathrm{n}=7)$ did not change 

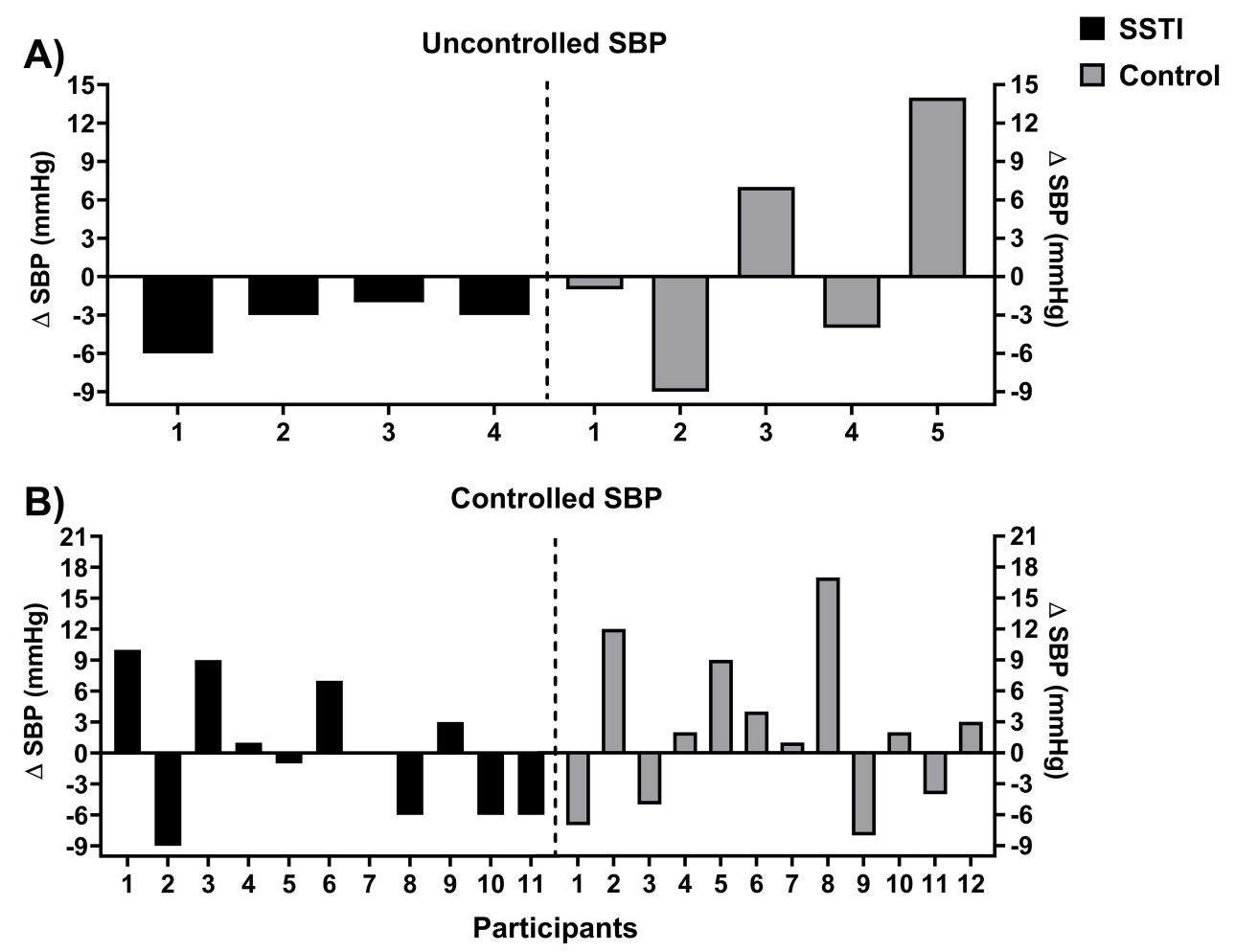

Figure 2 Individual responses of 24-hour ambulatory systolic blood pressure (SBP) in the participants from the self-selected training intensity (SSTI) and control groups with (B) controlled (ie SBP $<130 \mathrm{mmHg}$ ) and (A) uncontrolled (ie SBP $\geq 130 \mathrm{mmHg}$ ) blood pressure levels at baseline.

or reduced (from $\sim 6$ to $\sim 9 \mathrm{mmHg}$ ) the 24-hour systolic BP, and $36.3 \%(n=4)$ showed an increase. For the control group, $41.7 \%(n=5)$ did not change or reduced (from $\sim 3$ to $\sim 8 \mathrm{mmHg})$ the 24 -hour systolic BP and $58.3 \%(\mathrm{n}=7)$ showed an increase.

Table 4 shows the values of six-minute walking test performance at baseline and following the 8 weeks of intervention in the SSTI and control groups. Intention-totreat and per-protocol analysis revealed a significantly greater performance in the SSTI compared to the control group $(\mathrm{p}<0.05)$.

\section{Discussion}

The main findings of this study were: i) no changes in ambulatory BP occurred following 8 weeks of SSTI; ii) participants from the SSTI group improved their 6MWT performance; iii) the participants exercised at a moderate intensity (40-59\% of HR reserve) over the eight-week intervention period, reported the SSTI sessions as "light" (RPE 11) and "pleasant" (FS 3.5), and showed an attendance rate $>90 \%$. Thus, our hypothesis that 8 weeks of SSTI would elicit a reduction on ambulatory BP was not confirmed. To the best of our knowledge, this is the

Table 4 Six-Minute Walking Test Performance at Baseline and Following Eight Weeks of Intervention in the Self-Selected Training Intensity and Control Groups

\begin{tabular}{|c|c|c|c|c|c|c|c|c|}
\hline & \multicolumn{2}{|c|}{$\begin{array}{l}\text { SSTI } \\
\text { ITT (n=20); PP (n=I5) }\end{array}$} & \multirow[t]{2}{*}{ ES } & \multicolumn{2}{|c|}{$\begin{array}{l}\text { Control } \\
\text { ITT }(n=20) ; \text { PP }(n=\mid 7)\end{array}$} & \multirow[t]{2}{*}{ ES } & \multirow[t]{2}{*}{$\beta(95 \%$ Wald Cl) } & \multirow[t]{2}{*}{ P-value } \\
\hline & Baseline & 8 Week & & Baseline & 8 Week & & & \\
\hline $\begin{array}{l}\text { Intention-to-Treat } \\
\text { Distance }(\mathrm{m})\end{array}$ & $467 \pm 45$ & $482 \pm 46$ & 0.49 & $465 \pm 67$ & $459 \pm 76$ & 0.43 & 20 (I to 40$)$ & 0.040 \\
\hline $\begin{array}{l}\text { Per protocol } \\
\text { Distance }(\mathrm{m})\end{array}$ & $472 \pm 48$ & $491 \pm 47$ & 0.55 & $454 \pm 62$ & $447 \pm 72$ & 0.19 & 26 (2 to 50$)$ & 0.031 \\
\hline
\end{tabular}

Notes: Values expressed as mean \pm SD and parameter estimates $(\beta)$ and $95 \%$ Wald confidence interval $(\mathrm{Cl})$. ES: Cohen's $d_{z}$ effect size $(<0.50$, small; 0.50 to 0.79 , medium; and $\geq 0.80$, large).

Abbreviations: ITT, intention-to-treat analysis; PP, per-protocol analysis; SSTI, self-selected training intensity group. 
first randomized controlled trial that has investigated the short-term chronic effect of a SSTI intervention on ambulatory BP in hypertensive older women.

Our research group has previously shown that a single 30 -minute SSTI session reduced $\sim 6 \mathrm{mmHg}$ of ambulatory systolic BP in the first 6 hours post-exercise, which elicited a decrease in mean values of ambulatory BP in 20-hour and awake periods. ${ }^{16}$ Therefore, a SSTI session elicits an ambulatory $\mathrm{PEH}$, which plays a role in attenuating the overload imposed on the cardiovascular system during daily activities in hypertensive individuals, and as a consequence can contribute to reduce the risk of acute cardiovascular events. The magnitude and duration of $\mathrm{PEH}$ elicited by a single exercise session provides insight into the future chronic BPlowering effect following a regular exercise training period. It is argued that repeated $\mathrm{PEH}$ over an exercise training period contributes to a chronic reduction in BP. ${ }^{17}$ This can occur as a simple superimposition of a temporal summation of this acute effect or as a combination with morphological and functional adaptation over time, ${ }^{34}$ for example, in the hemodynamic determinants of the BP (ie, cardiac output and peripheral vascular resistance) and its regulatory mechanisms.

However, similarly to what occurs with $\mathrm{PEH},{ }^{17}$ it seems that factors such as the participants' characteristics may influence the occurrence (or not) of a chronic BP-lowering effect and benefits on the mechanisms related to the hemodynamic determinants of the BP control. Considering both intention-to-treat and per-protocol analyses, no significant changes in ambulatory BP (ie, 24-hour, awake and asleep periods) occurred following 8 weeks of SSTI. Regarding the baseline BP levels of the participants, 70\% had controlled 24-hour ambulatory BP levels $(\sim 115 / 66 \mathrm{mmHg})$ and $30 \%$ had uncontrolled BP levels ( 143/78 $\mathrm{mmHg})$. Previous meta-analyses clearly showed that the BP level pre-intervention influences the magnitude of BP-lowering effect, both for resting ${ }^{35}$ and ambulatory BP. ${ }^{31}$ Cornelissen et $\mathrm{al}^{35}$ and Sosner et $\mathrm{al}^{31}$ showed that the magnitude of a BPlowering effect following an aerobic exercise training intervention is greater in individuals with higher BP levels compared to those with optimal BP levels. A recent network meta-analysis involving more than 10,000 participants in exercise training interventions confirmed these previous findings. ${ }^{8}$ Considering the individual responses of those who concluded the 8 weeks of SSTI, all participants with uncontrolled BP level in our study showed a reduction in the 24-hour systolic BP (from $\sim 2$ to $\sim 6 \mathrm{mmHg}$ ). In addition, $63.7 \%(n=7)$ of those with controlled BP level did not change or reduced their 24-hour systolic BP. This scenario was different for the control group, considering both participants with uncontrolled (40\% increased BP; $n=2)$ and controlled (58.3\% increased BP; $\mathrm{n}=7$ ) BP level. Our findings seem to support previous studies that have shown a more consistent BP-lowering effect following an exercise training intervention in individuals with higher BP levels. $^{31,35}$ Thus, we do not rule out the possibility that a short-term SSTI-based intervention elicits better BP control in hypertensive older women with uncontrolled BP levels. Future randomized controlled trials, including only hypertensive older individuals with uncontrolled BP levels, should be conducted in order to confirm (or not) this assumption.

A reduction of $5 \mathrm{mmHg}$ in BP unequivocally decreases the risk of cardiovascular diseases and mortality. ${ }^{29,30}$ However, it should be noted that a reduction in BP of a lower magnitude than $5 \mathrm{mmHg}$ may be clinically meaningful. For example, a $2 \mathrm{mmHg} \mathrm{BP}$ reduction is sufficient to reduce the mortality risk by stroke in $6 \%$, coronary heart disease in $4 \%$, and all-cause mortality in 3\%. ${ }^{36}$ As all participants from the SSTI group with uncontrolled BP had a reduction in 24-hour systolic BP of at least $2 \mathrm{mmHg}$, it seems reasonable to assume that these individuals improved their cardiovascular disease risk profile following the 8 weeks of the exercise training intervention. In addition, the participants accumulated 150 minutes per week of moderate-vigorous aerobic exercise from week five to eight of the SSTI, meeting the threshold recommended by the American College of Sports Medicine ${ }^{7}$ and most hypertension guidelines. $^{37,38}$ A longitudinal study conducted by Brown et $\mathrm{al}^{39}$ involving 10,665 middle-aged and older adults with a mean follow-up of 8.6 years revealed that those who were inactive with controlled BP levels among medicated hypertensive individuals had a $42 \%$ higher risk of all-cause mortality than their active peers. Inactive individuals with uncontrolled BP levels had a 55\% higher risk of all-cause mortality than the active individuals with controlled BP levels. Active individuals with uncontrolled BP levels showed a trend $(\mathrm{p}=0.08)$ of $17 \%$ higher risk of all-cause mortality compared to their peers with controlled BP levels. These data reinforce the protective role of regular exercise against all-cause mortality in medicated hypertensive middle-aged and older individuals, independent of their BP status (ie, controlled or uncontrolled). The SSTI, which is a simple, low-cost, and feasible approach for "real world" settings, may be a viable way to induce an active lifestyle in 
hypertensive older individuals and potentially reduce their risk of mortality.

It was observed an increase of 6MWT performance (the secondary outcome of the present study) in the SSTI group compared to control group following 8 weeks of intervention. This result suggests an improvement in cardiorespiratory fitness and, as a consequence, a potential cardioprotective benefit in the participants from the SSTI group. Previous studies have shown a strong correlation between the distance covered in the 6MWT and the maximal oxygen uptake obtained from a maximal cardiopulmonary exercise, which is a "gold standard" method to determine cardiorespiratory fitness in different populations, including older adults with and without known cardiovascular disease. ${ }^{40-42}$ In the present study, the participants exercised within the moderate-intensity domain (ie, $40-59 \%$ of HR reserve) over the 8 weeks of SSTI. Moderate-intensity aerobic exercise is recommended to improve cardiorespiratory fitness in older adults, ${ }^{43}$ which seems to explain the improvements in the 6MWT performance observed in the SSTI group. Future studies should determine the effect of SSTI on cardiorespiratory fitness using a cardiopulmonary exercise test, given the clinical importance of this outcome for the cardiovascular health; ie, an increase of 1 metabolic equivalent (MET) in cardiorespiratory fitness is associated with a $10 \%$ to $20 \%$ decrease in mortality rates. ${ }^{25}$

The participants showed a consistent HR $(52 \pm 10 \%$ of HR reserve), RPE (11 \pm 1$)$, and affective valence (3.4 \pm 1.1$)$ during the exercise sessions over the 8 weeks of SSTI. Overall, these responses indicate that the inactive hypertensive older women exercised within a moderate intensity and reported the exercise sessions as "light" and "pleasant". In addition, they showed a high attendance rate to the exercise sessions (91.7\%). These findings confirm the results of two previous studies from our research group which showed similar HR, RPE, and affective valence patterns during a single $^{16}$ and two SSTI sessions ${ }^{15}$ separated by a one-week interval. The consistent positive affective valence over the SSTI sessions may be partially attributed to the magnitude of the metabolic stress imposed on the organism of the participants. Considering the HR and RPE responses, the participants probably self-selected an exercise intensity close but below their ventilatory or lactate threshold or the onset of blood lactate accumulation, which is consistent with the dual-mode theory. This theory postulates that when the individuals exercise at a moderate intensity (below the ventilatory threshold) they report a positive affective valence. ${ }^{13}$ In addition, the outdoor environment also contributes to a more positive affective valence due to a more dissociative attentional focus during exercise. ${ }^{44}$ Taken together, SSTI outdoors seems to be a feasible approach to encourage a more active lifestyle in hypertensive older women. From a practical perspective, SSTI should be considered in primary care and clinical settings both for physical activity counseling interventions and structured exercise programs delivered to hypertensive older women.

This study has limitations that should be mentioned. First, most participants from our sample had well-controlled ambulatory BP levels, which limits understanding the effect of SSTI in hypertensive older women with uncontrolled BP levels. Second, although we instructed the participants to maintain the physical activity pattern in their daily routine, we did not objectively assess this aspect. Therefore, it is not possible to determine whether the SSTI group or control group changed their physical activity level over the 8 weeks of intervention. Third, the participants were not familiarized with the ambulatory BP device, which can have elicited an alert reaction to wearing this device for the first time. ${ }^{45}$

\section{Conclusion}

Eight weeks of SSTI does not reduce 24-hour ambulatory BP in medicated hypertensive older women. However, SSTI increases the 6MWT performance in these individuals, which suggests improvement in cardiorespiratory fitness and a potential cardioprotective effect. Hypertensive older women self-select an exercise intensity within the recommendations of the clinical hypertension guidelines (ie, moderate-vigorous intensity), report the exercise sessions as "light" and "pleasant" and show a high attendance rate to the SSTI intervention. Future studies should consider investigating the long-term effect of SSTI on ambulatory BP and other cardiovascular- and fitness-related outcomes in hypertensive older patients.

\section{Data Sharing Statement}

The authors share the original database regarding outcomes of this study in the public repository for undetermined time, in order to independent verification of research results. The database can be found in: http://dx. doi.org/10.17632/ym2wtvmd7g.1.

\section{Funding}

JS was supported by the Brazilian National Council for Scientific and Technological Development (CNPq). RAVB, GADM, LLPC, and LFFJ are supported by the Brazilian Coordination for the Improvement of Higher Education Personnel (CAPES). 


\section{Disclosure}

The authors report no conflicts of interest in this work.

\section{References}

1. Whelton PK, Carey RM, Aronow WS, et al. ACC/AHA/AAPA/ABC/ ACPM/AGS/APhA/ASH/ASPC/NMA/PCNA guideline for the prevention, detection, evaluation, and management of high blood pressure in adults: executive summary: a report of the American College of Cardiology/American Heart Association Task F. Hypertens (Dallas, Tex 1979). 2017;2018(71):1269-1324.

2. Zhou B, Bentham J, Di Cesare M, et al. Worldwide trends in blood pressure from 1975 to 2015: a pooled analysis of 1479 populationbased measurement studies with $19 \cdot 1$ million participants. Lancet. 2017;389:37-55. doi:10.1016/S0140-6736(16)31919-5

3. Mills KT, Bundy JD, Kelly TN, et al. Global disparities of hypertension prevalence and control: a systematic analysis of population-based studies from 90 countries. Circulation. 2016;134:441-450. doi:10.1161/ CIRCULATIONAHA.115.018912

4. Benjamin EJ, Virani SS, Callaway CW, et al. Heart disease and stroke statistics-2018 update: a report from the American Heart Association. Circulation. 2018;137. doi:10.1161/CIR.000000 0000000558

5. Reichert FF, Azevedo MR, Breier A, et al. Physical activity and prevalence of hypertension in a population-based sample of Brazilian adults and elderly. Prev Med (Baltim). 2009;49:200-204. doi:10.1016/j.ypmed.2009.06.009

6. Weber MA, Schiffrin EL, White WB, et al. Clinical practice guidelines for the management of hypertension in the community: a statement by the American Society of Hypertension and the International Society of Hypertension. J Clin Hypertens (Greenwich). 2014;16:14-26. doi:10.1111/jch. 12237

7. Pescatello LS, MacDonald HV, Lamberti L, et al. Exercise for hypertension: a prescription update integrating existing recommendations with emerging research. Curr Hypertens Rep. 2015;17:87. doi:10.1007/s11906-015-0600-y

8. Naci H, Salcher-Konrad M, Dias S, et al. How does exercise treatment compare with antihypertensive medications? A network meta-analysis of 391 randomised controlled trials assessing exercise and medication effects on systolic blood pressure. Br J Sports Med. 2019;53:859-869. doi:10.1136/bjsports-2018-099921

9. Bueno DR, de Fátima Nunes Marucci $M$, Gobbo LAet al. Expenditures of medicine use in hypertensive/diabetic elderly and physical activity and engagement in walking: cross sectional analysis of SABE survey. BMC Geriatr. 2017;17:70. doi:10.1186/s12877-0170437-0

10. Ekkekakis P. Let them roam free? Physiological and psychological evidence for the potential of self-selected exercise intensity in public health. Sport Med. 2009;39:857-888. doi:10.2165/11315210000000000-00000

11. Lind E, Joens-Matre RR, Ekkekakis P. What intensity of physical activity do previously sedentary middle-aged women select? Evidence of a coherent pattern from physiological, perceptual, and affective markers. Prev Med (Baltim). 2005;40:407-419. doi:10. 1016/j.ypmed.2004.07.006

12. Ekkekakis P, Lind E. Exercise does not feel the same when you are overweight: the impact of self-selected and imposed intensity on affect and exertion. Int $J$ Obes (Lond). 2006;30:652-660. doi:10.1038/sj.ijo.0803052

13. Ekkekakis P, Parfitt G, Petruzzello SJ. The pleasure and displeasure people feel when they exercise at different intensities decennial update and progress towards a tripartite rationale for exercise intensity prescription. Sport Med. 2011;41:641-671. doi:10.2165/11590 680-000000000-00000
14. Smith AE, Eston R, Tempest GD, et al. Patterning of physiological and affective responses in older active adults during a maximal graded exercise test and self-selected exercise. Eur J Appl Physiol. 2015;115:1855-1866.

15. Sócrates J, Macêdo GAD, Costa IBB, et al. Self-selected exercise intensity for inactive hypertensive older women: a pilot study. Rev Bras Atividade Física Saúde. 2019;24:1-9. doi:10.12820/ Rbafs.24e0084

16. Costa IBB, Schwade D, Macêdo GAD, et al. Acute antihypertensive effect of self-selected exercise intensity in older women with hypertension: a crossover trial. Clin Interv Aging. 2019;14:1-12. doi: $10.2147 /$ CIA.S207254

17. Brito LC, Fecchio RY, Peçanha T, et al. Postexercise hypotension as a clinical tool: a 'single brick' in the wall. $J$ Am Soc Hypertens. 2018;12:e59-e64. doi:10.1016/j.jash.2018.10.006

18. Malachias MVB, Gomes MAM, Nobre F, et al. 7th Brazilian Guideline of Arterial Hypertension: chapter 2 - diagnosis and classification. Arq Bras Cardiol. 2016;107:7-13. doi:10.5935/ abc. 20160152

19. Pereira DS, Dias RC; Lustosa LPT and cultural adaptation of the MLTAQ in community-dwelling older people, et al.. Translation and cultural adaptation of the Minnesota Leisure Time Activities Questionnaire in community-dwelling older people. Geriatr Gerontol. 2011;5:57-65.

20. Tudor-Locke C, Hatano Y, Pangrazi RP, et al. Revisiting 'how many steps are enough?'. Med Sci Sports Exerc. 2008;40:S537-43. doi:10.1249/MSS.0b013e31817c7133

21. Thomas S, Reading J, Shephard RJ. Revision of the Physical Activity Readiness Questionnaire (PAR-Q).. Can J Sport Sci. 1992;17 (4):338-345.

22. Borg G. The Borg RPE Scale. In: Borg G. Borg's Perceived Exertion and Pain Scales. Champaign, IL: Human Kinetics; 1998:29-38.

23. Riebe D, Ehrman JK, Liguori G, et al. ACSM's Guidelines for Exercise Testing and Prescription. 10th. Philadelphia: Wolters Kluwer Health; 2018.

24. Rikli RE, Jones CJ. Development and validation of a functional fitness test for community-residing older adults. $J$ Aging Phys Act. 1999;7:129-161. doi:10.1123/japa.7.2.129

25. Ross R, Blair SN, Arena R, et al. Importance of assessing cardiorespiratory fitness in clinical practice: a case for fitness as a clinical vital sign: a scientific statement from the American Heart Association. Circulation. 2016;134:e653-e699. doi:10.1161/CIR.0000000000000461

26. Sperandio EF, Arantes RL, Matheus AC, et al. Intensity and physiological responses to the 6-minute walk test in middle-aged and older adults: a comparison with cardiopulmonary exercise testing. Brazilian J Med Biol Res. 2015;48:349-353. doi:10.1590/1414$431 \times 20144235$

27. Nobre F, Mion Júnior D, Gomes M, et al. 6th guideline for ambulatory blood pressure monitoring and 4th guideline for household blood pressure monitoring. Arq Bras Cardiol. 2018;110:1-29. doi:10.5935/ abc. 20180012

28. Hardy CJ, Rejeski WJ. Not what, but how one feels: the measurement of affect during exercise. J Sport Exerc Psychol. 1989;11:304-317. doi:10.1123/jsep.11.3.304

29. Bundy JD, Li C, Stuchlik P, et al. Systolic blood pressure reduction and risk of cardiovascular disease and mortality: a systematic review and network meta-analysis. JAMA Cardiol. 2017;2:775-781. doi:10.1001/jamacardio.2017.1421

30. Ettehad D, Emdin CA, Kiran A, et al. Blood pressure lowering for prevention of cardiovascular disease and death: a systematic review and meta-analysis. Lancet (London, England). 2016;387:957-967. doi:10.1016/S0140-6736(15)01225-8

31. Sosner P, Guiraud T, Gremeaux V, et al. The ambulatory hypotensive effect of aerobic training: a reappraisal through a meta-analysis of selected moderators. Scand J Med Sci Sports. 2017;27:327-341. doi:10.1111/sms.12661 
32. Lakens D. Calculating and reporting effect sizes to facilitate cumulative science: a practical primer for t-tests and ANOVAs. Front Psychol. 2013;4. doi:10.3389/fpsyg.2013.00863.

33. Cohen J. Statistical Power Analysis for the Behavioral Sciences. 2nd ed. New Jersey: Lawrence Erlbaum; 1988.

34. Lucas da Nobrega AC. The subacute effects of exercise: concept, characteristics, and clinical implications. Exerc Sport Sci Rev. 2005;33:84-87. doi:10.1097/00003677-200504000-00005

35. Cornelissen VA, Smart NA. Exercise training for blood pressure: a systematic review and meta-analysis. J Am Heart Assoc. 2013;2: e004473-e004473. doi:10.1161/JAHA.112.004473

36. Whelton PK, He J, Appel LJ, et al. Primary prevention of hypertension: clinical and public health advisory from The National High Blood Pressure Education Program. JAMA. 2002;288:1882-1888. doi:10.1001/jama.288.15.1882

37. Eckel RH, Jakicic JM, Ard JD, et al. AHA/ACC guideline on lifestyle management to reduce cardiovascular risk: a report of the American College of Cardiology/American Heart Association Task Force on Practice Guidelines. Circulation. 2013;2014(129):S76-99.

38. Mancia G, Fagard R, Narkiewicz K, et al. ESH/ESC Guidelines for the management of arterial hypertension: the Task Force for the management of arterial hypertension of the European Society of Hypertension (ESH) and of the European Society of Cardiology (ESC). J Hypertens. 2013;2013(31):1281-1357. doi:10.1097/01. hjh.0000431740.32696.cc
39. Brown RE, Riddell MC, Macpherson AK, et al. The joint association of physical activity, blood-pressure control, and pharmacologic treatment of hypertension for all-cause mortality risk. Am J Hypertens. 2013;26:1005-1010. doi:10.1093/ajh/hpt063

40. Araújo CO, Makdisse MRP, Peres PAT, et al. Different patterns for the 6-minute walk test as a test to measure exercise ability in elderly with and without clinically evident cardiopathy. Arq Bras Cardiol. 2006;86:198-205. doi:10.1590/S0066-782X2006000300007

41. Roul G, Germain P, Bareiss P. Does the 6-minute walk test predict the prognosis in patients with NYHA class II or III chronic heart failure? Am Heart J. 1998;136:449-457. doi:10.1016/S0002-8703(98)70219-4

42. Zugck C, Krüger C, Dürr S, et al. Is the 6-minute walk test a reliable substitute for peak oxygen uptake in patients with dilated cardiomyopathy? Eur Heart J. 2000;21:540-549. doi:10.1053/ euhj.1999.1861

43. Piercy KL, Troiano RP, Ballard RM, et al. The physical activity guidelines for Americans. JAMA. 2018;320:2020-2028.

44. Krinski K, Machado DGS, Lirani LS, et al. Let's walk outdoors! selfpaced walking outdoors improves future intention to exercise in women with obesity. J Sport Exerc Psychol. 2017;39:145-157. doi:10.1123/jsep.2016-0220

45. Ash G, Walker T, Olson K, et al. Reproducibility of ambulatory blood pressure changes from the initial values on two different days. Clinics. 2013;68:1509-1515. doi:10.6061/clinics/2013(12)06
Clinical Interventions in Aging

\section{Publish your work in this journal}

Clinical Interventions in Aging is an international, peer-reviewed journal focusing on evidence-based reports on the value or lack thereof of treatments intended to prevent or delay the onset of maladaptive correlates of aging in human beings. This journal is indexed on PubMed Central, MedLine, CAS, Scopus and the Elsevier

\section{Dovepress}

Bibliographic databases. The manuscript management system is completely online and includes a very quick and fair peer-review system, which is all easy to use. Visit http://www.dovepress.com/ testimonials.php to read real quotes from published authors. 